\title{
Embedded Commissioning for Building Design
}

\author{
O. Akin, M.T. Turkaslan-Bulbul \& I. Gursel \\ School of Architecture Carnegie Mellon University, Pittsburgh, USA
}

\author{
J.H. Garrett Jr, B. Akinci, H. Wang \\ Department of Civil and Environmental Engineering Carnegie Mellon University, Pittsburgh, USA
}

ABSTRACT: Building Commissioning has a broad scope that extends to all phases of building delivery. We view commissioning as a building delivery embedded process that persistently verifies and validates design intent throughout the building lifecycle process. In the building lifecycle approach, buildings are considered to have cradle-to-grave life spans. They are modeled through a variety of different developmental phases. In this research project, we intend to build the necessary theory and tools to support the embedded commissioning process as a co-function of building lifecycle.

\section{INTRODUCTION}

Building commissioning is an important new area of practice and research in the industry. It has emerged, during the last 25 years, as the central phase of building delivery that is responsible for verifying design intent. Currently, it is rapidly becoming the performance verification tool in HVAC design and LEED (Leadership in Energy and Environmental Design) certification in the USA.

Building commissioning is a multi-phase process that ensures the interacting systems in a building are properly installed and operating. In the early phases of facility design, commissioning is concerned with whether the program and the design are delivering the owner's desired functionality. During the construction process, commissioning is concerned with ensuring that the performance of the selected building equipment agrees with the design specifications and delivers the intended functionality. The process of building commissioning tends to generate large amounts of data, much of which needs to be shared across other facility delivery phases.

We view commissioning as a building delivery embedded process that persistently verifies and validates design intent throughout the building lifecycle. The Embedded Commissioning Model (ECM), which is described in this paper, combines the processes of commissioning and building life-cycle in order to provide a framework for managing the information exchange between them. Here, the role of commissioning is to complement each of the lifecycle phases and their interactions through timely building system evaluation.

The primary objective of our study is to investigate the computability of Embedded Commissioning (EC) for HVAC systems. Our approach focuses on exploring the representational needs of the EC process and the management of EC data. Here, we concentrate on how the EC process works? What kind of information is produced; and what type of attributes can be defined? The output of this study is used to develop a proof of concept prototype software that supports the decision making process in EC.

\section{RESEARCH BACKGROUND}

\subsection{History}

The term commissioning has originated from the naval practices. Commissioning ceremony is a sign that the ship is accepted as an operating unit of the navy. By breaking the commissioning pennant the ship is put into the responsibility of the commanding officer who together with the ship's crew has the task of making and keeping her ready for any service required during peace or war. Prior to commissioning, the newly-launched vessel must pass some tests before she is considered complete and ready to be authorized as a commissioned ship. The new ship goes through several sea trials during which deficiencies that need correction are uncovered. The crew and the ship must function in total harmony for maximum effectiveness and efficiency (Reilly 1975).

The association between ships and buildings is not new but commissioning was introduced into the building industry only during 1977. Public Works Canada is the first organization who started to use commissioning in project delivery. Then in 1981 Disney Inc. issued a comprehensive commissioning program in the design, construction and start-up of its Epcot theme park.

In the United States of America, formal work on the commissioning process began in 1984 when the American Society of Heating Refrigerating and Air-Conditioning Engineers (ASHRAE) formed Commissioning Guideline Committee. The task of the committee was to define a process which guarantees that fully functioning buildings were turned over to the building owners. The motivation for the ASHRAE Commissioning Committee was the growing number of complaints about unmanageable HVAC systems, increasing operation expenses, decreasing comfort levels, and uneducated operations and maintenance staff who did not understand how to maintain or operate new buildings. After its foundation, the ASHRAE commissioning committee published two guidelines. The original guideline was announced in 1989 and an updated version has been published in 1996 (Guideline 1996-1).

After the announcement of ASHRAE Commissioning Guidelines, commissioning practice started to draw attention from various areas. University of Wisconsin, Madison offered commissioning courses and University of Michigan established a facilities Proceedings of the Fourth International Conference for Enhanced Building Operations, Paris, France, October 18-19, 2004 
evaluation and commissioning group. In 1993 first National Conference on Building Commissioning (NCBE) wass held and National Environmental Balancing Bureau (NEBB) developed a commissioning providers' certification program. After 1993 a range of governmental and private organizations started commissioning practices and issued regulations or guidelines. In 1998 US Green Building Council added commissioning to Leadership in Energy and Environmental Design (LEED) criteria. Finally, in 1999 the Building Commissioning Association (BCA) was established.

\subsection{Definition}

ASHRAE defines commissioning as the process of ensuring that systems are designed, installed, functionally tested and capable of being operated and maintained to perform in conformity with the design intent (Guideline 1-1996). Commissioning is a systematic approach. It starts with the programming phase and ends when the building is turned over to the owner. Most commissioning companies also provide a one or two year guarantee phase after the building is occupied. During the commissioning period the aim is to ensure and verify, with documentation, that all building systems perform in the way that they were intended and the operating and maintenance staff is trained according to the owner's operational needs.

Commissioning is occasionally confused with testing, adjusting and balancing (TAB) process or the punch list inspection. The latter is a physical examination done before a building is turned over to its owner. It is a one day process at the end of which a list of missing elements are identified such as "door stops are missing" or "vinyl base needed in the emergency exit stairway." TAB is a more complex process than punch list inspections. It measures air and water flows in building's HVAC systems. Punch list inspections and TAB process mainly focus on items that are important to get regulatory occupancy permits and opening the building.

Commissioning covers a much broader scope of work than these inspections. It necessitates functional testing to determine how well building systems perform together and verifies the results of TAB reports. Applying functional tests to individual equipment and whole building systems also help determine whether the tested item meets operational goals or if it needs modification to increase its efficiency and effectiveness.

This standard definition of commissioning refers to a process which starts at the building's design phase and ends when the building is turned over to the owner. However existing practice of building construction does not require building owners to hire a commissioning professional at the beginning of the project. The commissioning processes can be adapted to any phase during the lifecycle of a building.

\section{PROBLEM STATEMENT}

The conventional building delivery process begins with the recognition of the need for physical intervention and concludes by the eventual decommissioning of a building having gone through a set of predefined stages (Davis et. al., 1974). We use the term "building lifecycle" as a reference to an expanded and improved version of such conventional delivery models.

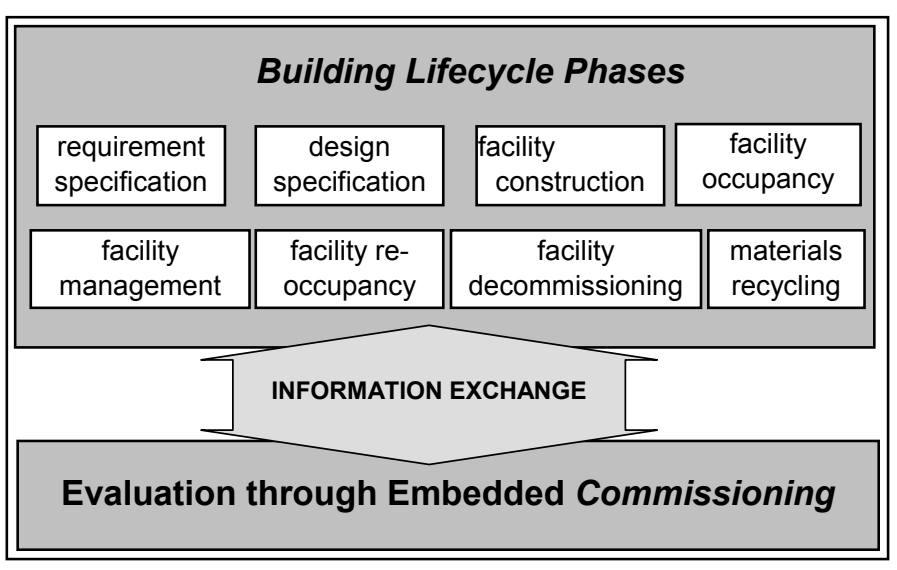

Figure 1. The Embedded Commissioning Model
In the building lifecycle approach, buildings are considered to have cradle-to-grave life spans. They are modeled through a variety of different developmental phases, rather than a set, lockstep procedure. These phases include: requirement specification, design specification, facility construction, facility (de)commissioning, facility (re-)occupancy, facility management, and materials recycling. They can take place, iteratively, in smaller or larger process cycles, at anytime during a building project's lifetime.

The role of Embedded Commissioning (EC) in this cycle is to accompany each of these phases and their interactions with timely building system evaluation. Figure 1 shows the function of Embedded Commissioning Model (ECM), which mediates between commissioning and building life-cycle for managing the information exchange between them.

For instance, facility construction normally would begin once design specification is complete. Commissioning, at this point, would serve as the evaluation aspect of the construction process, periodically verifying the accuracy of what is being constructed against available specifications, whether these are of a design or requirement type. In response to this evaluation, the construction process would either continue as planned or be modified. This kind of feedback cycle is imaginable for all eight phases of the lifecycle model shown in Figure 1. Furthermore, by considering commissioning as a parallel and interconnected activity we intend to realize its potential impact for the entire ECM for all phases of the building lifecycle.

In order to consider the implications of this approach we will analyze the role of EC in the "design specification" phase, just one of the eight shown in Figure 1, as a descriptive example. 
There are three major factors that inform the potential impact of commissioning on design specification: decision complexity, system integration and information seams of the lifecycle model.

\subsubsection{Decision Complexity}

In the design specification phase, particularly in the earlier stages, one of the most difficult challenges is to manage complexity. This complexity arises from the inherent interdependence between different design decisions (Akin 1978).

For instance, determining the number of floors in a hospital building, the distribution of the hospital functions on each floor, the organization of circulation into various configurations, can constitute important design decisions. Deciding to create a tall hospital building will impose important constraints on the circulation diagram on each floor. Depending on whether correlated programmatic functions can be located on the same floor, an appropriate circulation concept -- say, a linear, concentric, or satellite type -may be used. This in turn can determine the overall building configuration. In a real design situation there would be many more factors to consider, such as, cost, zoning limitations on building height, mechanical systems and their zoning requirements, and visual appearance.

Potentially, all of these factors may influence how such a building's spatial configuration decisions are made. Many of the consequences of such decisions would become apparent only when downstream decisions are made. Consequently, in order to manage the complexity factor, many iterations would be required to synchronize upstream decisions with down stream ones.

\subsubsection{System Integration}

Another aspect of the interaction of design and commissioning in the ECM is the unpredictable results of synergy that potentially exists between separately designed building subsystems. Structural systems can interfere with mechanical distribution. Lighting systems may cause extra cooling loads for the HVAC system. Circulation configurations may reveal unexpected privacy needs of occupants. Sometimes the only way to discover, let alone, resolve such conflicts, is to conduct elaborate simulations of proposed designs. It may even be plausible to accomplish this during the construction commissioning phase, that is, if the design allows for evolutionary construction stages planned to respond to the findings of persistent commissioning activities, as would be the case in ECM.

This suggests a design process which relies on persistent refinement and evolution of the design through evaluation and commissioning. While it would be impossible to do this with all aspects of a design problem, there are some subsystem performance values that are so difficult to predict during the early design process that it would even be desirable to postpone detailed decisions until after either design simulation or construction commissioning takes place. For example, some of these performance categories include: HVAC systems, acoustic systems, operable-window use patterns, vertical circulation use patterns, and emergency egress behavior.

\subsubsection{Information Seams in Building Delivery}

In the traditional building delivery process, some of these hot points of design refinement are managed through conventions of the design-delivery practice.

For instance, architects and their consulting engineers occasionally specify building designs only partially. They, intentionally, rely on the general contractors and their subs to provide the fabrication details for individual structural components or mechanical equipment. These are called shop drawings.

Furniture manufacturers and cabinet makers, often wait to obtain as-built drawings and dimensions before they design and fabricate furnishings. Similarly acoustic engineers rely on measurements and readings taken at the site. They also use mockups before finalizing their designs. It is not uncommon that paints and other finishes, even major cladding elements like brick and stone, are evaluated through samples installed at the site before final approvals are given.

Even when such sampling and measurements are made, it is not unusual to end up with insufficient data about existing conditions. Soil samples, for example, may not tell the entire story about what can be uncovered at the site once excavation takes place. At the time of demolition, existing structures on a site usually reveal more than just a few surprises.

The potential impact of some of these problems fit nicely into the professional know-how of specific building trades or the practices that apply to the individual stages of the delivery process. But others fall squarely at the boundaries of these stages or professional domains. This is precisely the reason why explicit protocols for information exchange between trades do exist. For example, it would be counter productive for the designers to prepare shop drawings for the steel work. Only the steel contractors would know with certainty how to meet the design requirements in the most economical and practical manner. One of the formal procedures for bridging such a seam is, in fact, the shop drawing preparation and approval process.

In practice, these procedures are imperfect. Many failures in buildings, some of which have achieved national notoriety, have been linked to information loss that has occurred at the shop drawing preparation and approval stage of building delivery -- John Hancock Tower, Boston MA; Citicorp Tower, NY, NY; Kansas City Hyatt Regency, Kansas MO (Akin 2001). 
The Embedded Commissioning Model (ECM), integrating commissioning within building lifecycle phases, is intended to address the need for continuous evaluation during not only the design specification phase (as addressed above) but also for all of the other phases of building lifecycle. This model is proposed as a framework to address problems of information management, such as the ones described in the previous section -- decision complexity, system integration and information seams.

The primary mechanism in this model is to execute each phase with the expectation that persistent evaluation will provide guidance for downstream decisions, based on ongoing commissioning measurements and simulations. We expect that this will significantly improve performance during all of the stages of the building lifecycle. For example in design specification phase two important improvements can be affected: (1) scoping design intentions more accurately based on greater downstream information obtained through the embedded commissioning process; and (2) phasing the entire scope of design intentions into smaller installments, in order to match them against the stages of ECM.

What we have outlined here for the design specification phase, we expect, holds for the other phases of building lifecycle, as well. The scope of our overall research program then is to identify process flow of embedded commissioning in building lifecycle phases and develop a data model that represents the information in it. These are going to be a base to build a computer assisted decision tool to enable all of the information exchange links between building lifecycle phases and the embedded commissioning activities.

In this paper, due to space limitations, we consider a much smaller portion of this agenda. In particular, we will describe a process model developed for commissioning HVAC systems during the facility programming, design, construction, acceptance and post-acceptance phases. We will then explain ECM data model that is developed to represent the information in our EC process model. At the end we will discuss a proof of concept application limited to the HVAC systems during the facility construction phase.

\section{APPROACH}

Our approach to investigate the computability of Embedded Commissioning is built on the formal representation of its process and data models.

\subsection{EC Process Model}

In almost every commissioning related source there is a description of how building commissioning should be done. Our aim for developing an EC flow chart was identifying a standardized process that we can use in our research. We used ASHRAE's commissioning description in its Guideline 1-1996 as our starting point. We have conducted a detailed study of the commissioning process described in that guideline. This revealed a well-structured methodology and yet promises to lead to a guide for people involved in building delivery, so as to achieve efficient, effective, and high quality HVAC systems. The ASHRAE guideline provides a model that shows commissioning activities step by step. However this model does not present the details of the flow of EC processes and their connections to different building delivery stages.

In order to develop a comprehensive model we conducted a detailed observation of an HVAC commissioning process of a new university dormitory at Carnegie Mellon University, in Pittsburgh, PA. We interviewed the commissioning team in order to primarily learn about a specific case of commissioning in detail so as to gain insights about the mechanics of the process. Our aim in this observation was to explore the larger context of EC and how it works in the normal. We analyzed the dormitory case so as to illuminate commissioning as a normative process. We are particularly interested in understanding the precise protocols and documents used in their inspections, tests and measurements, and the role of these documents in different phases of commissioning.

In our analysis of the ASHRAE guideline and dormitory commissioning, we described the EC process as an inclusive process flow illustrating every task, document and decision culled from all phases of commissioning (Akin et al. 2003). The flow chart is organized in the form of a design-bid-construct process which has five main phases: program phase, design phase, construction phase, acceptance phase and post-acceptance phase. All HVAC commissioning procedures are explained in relation to these building stages. Figure 2 is an example from our flowchart that shows the programming and design phases.

In our study, this detailed process model was important for three reasons. First, it showed us how people interact with each other during the commissioning process. Second, we could track what kind of documents are produced in this process and how they evolve throughout design. Third, it helped us to recognize the type of data used in EC, which needs to be identified and modeled.

\subsection{EC Data Model}

After completing the EC process model we started to model EC data. Data modeling for Embedded Commissioning has three steps. The first step is about identifying building commissioning data. In this phase, we looked at commissioning related information produced by different sources such as commissioning companies and organizations that publish commissioning guidelines or regulations. We compared these different groups of data through comparative analysis tables (CAT) and prepared a normalized data set. Next, we defined the structure of the data model that represents our normalized data set consistent with the needs of the EC process we defined in our EC process model. The third step, is about testing the developed model with existing building product models. We tested IFC's in order to see their support of the commissioning process and the possibility of data exchange in this context.

Proceedings of the Fourth International Conference for Enhanced Building Operations, Paris, France, October 18-19, 2004 

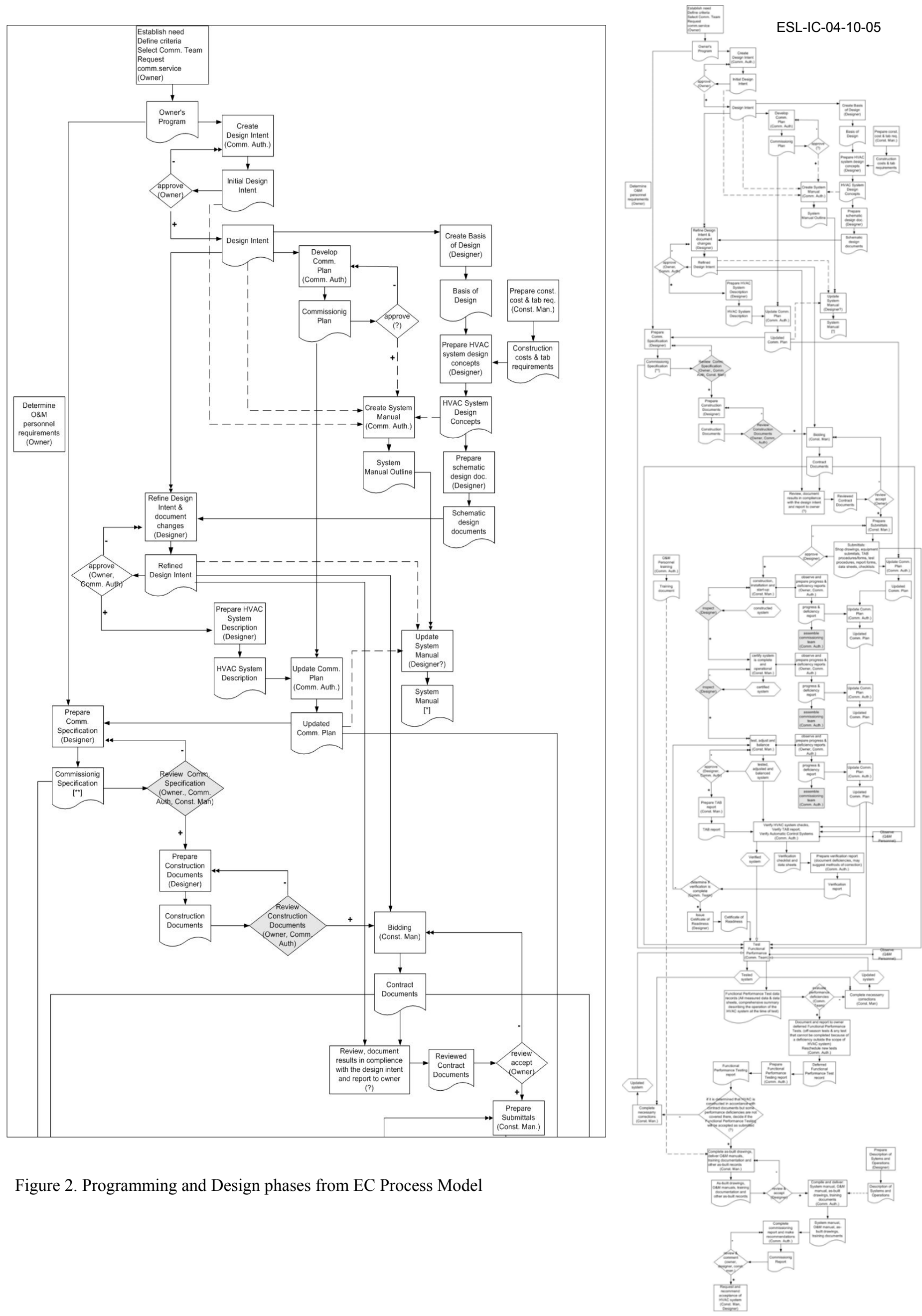

Figure 2. Programming and Design phases from EC Process Model 


\subsubsection{Identifying Commissioning Data}

While modeling the embedded commissioning process we have identified a group of commissioning data. In order to develop a normalized and consistent data set, we compared the data we have with other commissioning data produced by other sources. In our initial comparisons, we saw that commissioning data shows variations according to the source type. For accurate comparisons we identified four groups of commissioning sources: (i) data sheets of practicing commissioning agents; (ii) commissioning guidelines coming from organizations such as the American Society of Heating, Refrigerating and Air-Conditioning Engineers (ASHRAE), National Institute of Standards and Testing (NIST); (iii) equipment specifications of HVAC manufacturers such as Trane and Carrier; and (iv) the products of other research groups.

Table 1. Structure of Comparative Analysis Tables (CAT)

\begin{tabular}{|c|c|c|c|c|}
\hline Source 1 & Source 2 & Source 3 &.. & Source $n$ \\
\hline Fan capacity & Airflow (cfm) & $\begin{array}{l}\text { Fan CFM (total / } \\
\text { return / outside / } \\
\text { discharge) }\end{array}$ & & \\
\hline $\begin{array}{l}\text { Static pres- } \\
\text { sure }\end{array}$ & $\begin{array}{l}\text { Static pres- } \\
\text { sure (IN, W, } \\
\text { G) }\end{array}$ & $\begin{array}{l}\text { Static pressure } \\
\text { (elements) }\end{array}$ & & \\
\hline \multicolumn{5}{|l|}{$\ldots \ldots$} \\
\hline \multicolumn{5}{|l|}{$\begin{array}{l}\text { Fan full load } \\
\text { VFD Effi- } \\
\text { ciency }(\%)\end{array}$} \\
\hline \multicolumn{5}{|l|}{$\begin{array}{l}\text { Fan noise } \\
\text { class }\end{array}$} \\
\hline & & $\begin{array}{l}\text { Required toler- } \\
\text { ance }\end{array}$ & & \\
\hline & & Description & & \\
\hline & & $\ldots \ldots \ldots \ldots \ldots$ & & \\
\hline
\end{tabular}

Data coming from different sources are compiled into Comparative Analysis Tables (CAT). The comparison analysis tables are laid out according to various pieces of HVAC equipment, and each corresponding attribute is listed across the table to identify possible matches or similarities in that specific component's unique properties. By identifying prevalent attributes relevant to certain equipment, we were able to compile generalized properties of those components with the intention of using them to define the parameters of our model.

In CAT (Table 1) each column contains data from a specific source. For example, in "Fan Capacity", "Airflow (cfm)" and "Fan CFM (total / return / outside / discharge)"; coming from Sources 1, 2, and 3 respectively; refer to the same attribute of HVAC equipment, fan.

So they are all placed in the same row. However "Fan noise class" property from Source 1 does not have any match from Source 2 or Source 3. Respective cells left empty in those rows.

We selected three types of HVAC equipment according to the complexity of their attribute types. These types of equipment are the air filter, fan and Air Handling Unit (AHU). An air filter is a simple, single piece of equipment. It does not have too many variations and has a constant set of attributes for all air filter types. In comparison to the air filter, the fan is a more complex piece of equipment. According to its functionality, there may be hundreds of different varieties and fan attributes which change with respect to these variations. An AHU is the most complex piece of equipment that we have modeled, since it consist of other pieces of equipment such as coils, air filters, control sensors, supply and exhaust fans. Different combinations of these pieces of equipment can potentially create thousands of distinct AHU assemblies. Usually, the exact attributes of an AHU depends on the equipment types from which it is made.

We developed CATs for all three equipment types. The table produced for air filter was simple and it showed us that this comparison method is suitable for our work. The challenge in preparing a CAT of fans was to group fan types into reasonable categories that can be represented in our data model. For AHU it was not possible to put all information into one table. Instead we identified components of a medium AHU and compiled CATS for all of them. We identified nine components: air filters, fans, coils, sensors, humidifiers, ducts, dampers, pumps, VAV boxes, economizers.

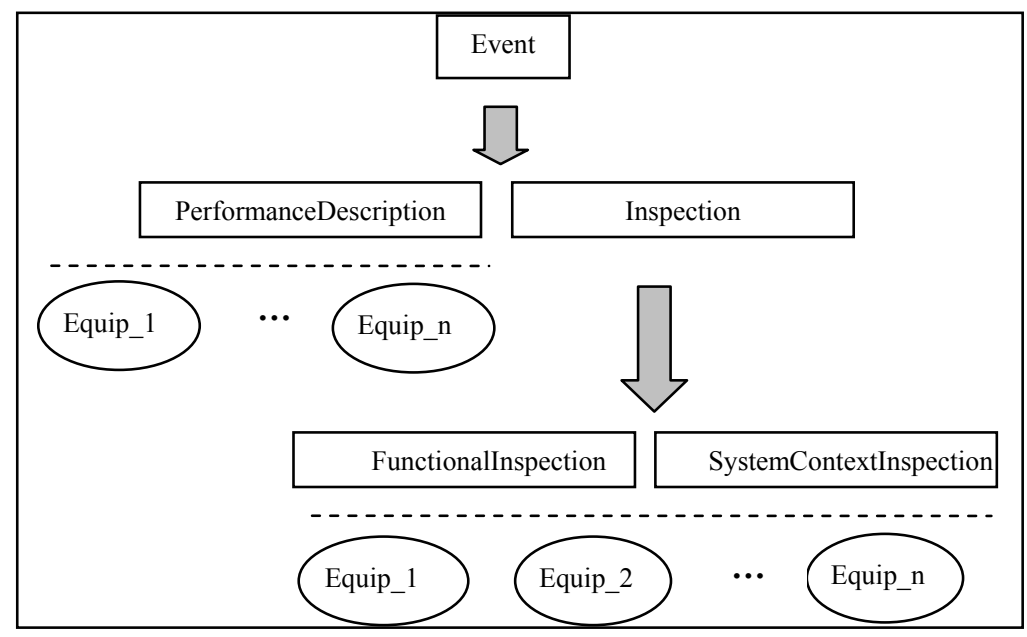

The challenge in describing commissioning data, in this fashion is to limit the number of sources to seminal ones. Every new source may bring a new attribute that has not been captured in the previous sources. This may be due to the type of source, type of commissioning practice or the type of building that has been commissioned. Our aim in identifying the commissioning data is to collect a reasonable number of attributes that refer to a comprehensive commissioning process. When we reach this point, new data additions will remain marginal. For air filters and fans we collected an adequate amount data.

\subsubsection{Structure of Data Model}

We developed our EC data model simultaneously with EC data identification, which allowed refining of the Figure 3. EC Data Model Structure data model as EC data were updated.

EC data modeling starts with understanding how the EC process works. For this, we relied on the model that we created previously. Our building commissioning data model is based on the assumption that there are three events in the building commissioning process that define EC data. 
These events are specification, system context inspection and functional inspection. Specification is done in the design phase and it describes the performance criteria. System context inspection and functional inspection are post-construction events and actual commissioning takes place through these inspections. System context inspection is a qualitative evaluation of the equipment and its content (e.g., "is the AHU properly supported") whereas functional inspection takes actual measurements and compares them with the values defined in the specification event.

The data in these events are organized in a hierarchical order from more general to specific. Figure 3 explains structure of our model according to this three event organization. The root of the model is Event class from which PerformanceDescription and Inspection classes inherit their properties. PerformanceDescription class represents the specification event and Inspection class represents the system context inspection and functional inspection events. They are specified as FunctionalInspection and SystemContextInspection classes in the third level. Specific information for every piece of equipment is added as branches to this structure. Figure 4 shows a part from EC model that is developed in UML, accordingly. Our aim in developing this data model has been to represent EC data in the real world. From our process model we know that real world commissioning exists under volatile information conditions. However our current data model represents only a stable group of data and any condition of unpredictability is a challenge for our model. Currently, we are working on refining our model for these volatile conditions. In the future, we will update our EC data model in a way to represent both predefined and yet-to-be-defined data.

\subsubsection{Testing How Well IFC Releases Support EC Model}

The adequacy IFC's is tested with developed EC data model in order to see their support of the commissioning process and explore the possibilities for data exchange. Due to space limitations we will only report the results of our testing. Further information can be found in other publications (Garrett et al, 2004).

For those attributes that do not have proper counterpart in the previous IFC models, IFC $2 \times 2$ provides a capability to exchange any customized data by using IfcPropertySet as a general container. Thus the IfcPropertySet class can be used as a general attribute container that holds any type of product property. However, IfcPropertySet only works within a single system because it does not have a public schema, which is the very protocol external applications expect during the data exchange.

Among all current EC entities, relationships and attributes in the EC model, about 30 percent of those data items can be fully matched to the entities in the most recent release of the IFC data exchange standard, R2x2. For example, in IFC R2x2, Equipment entity, which stands for HVAC equipment, is represented by IfcElement and Event entity, which stands for commissioning activity, is expressed by IfcTask. IfcRelAssignToProcess entity is used to represent three kinds of relationships between Equipment and Event entities: functional_inspection, specification, and system_context_inspection. For the high level classes, e.g. Equipment, Event and their direct subclasses, more than $60 \%$ of attributes can be represented by standard IFC classes and IFC

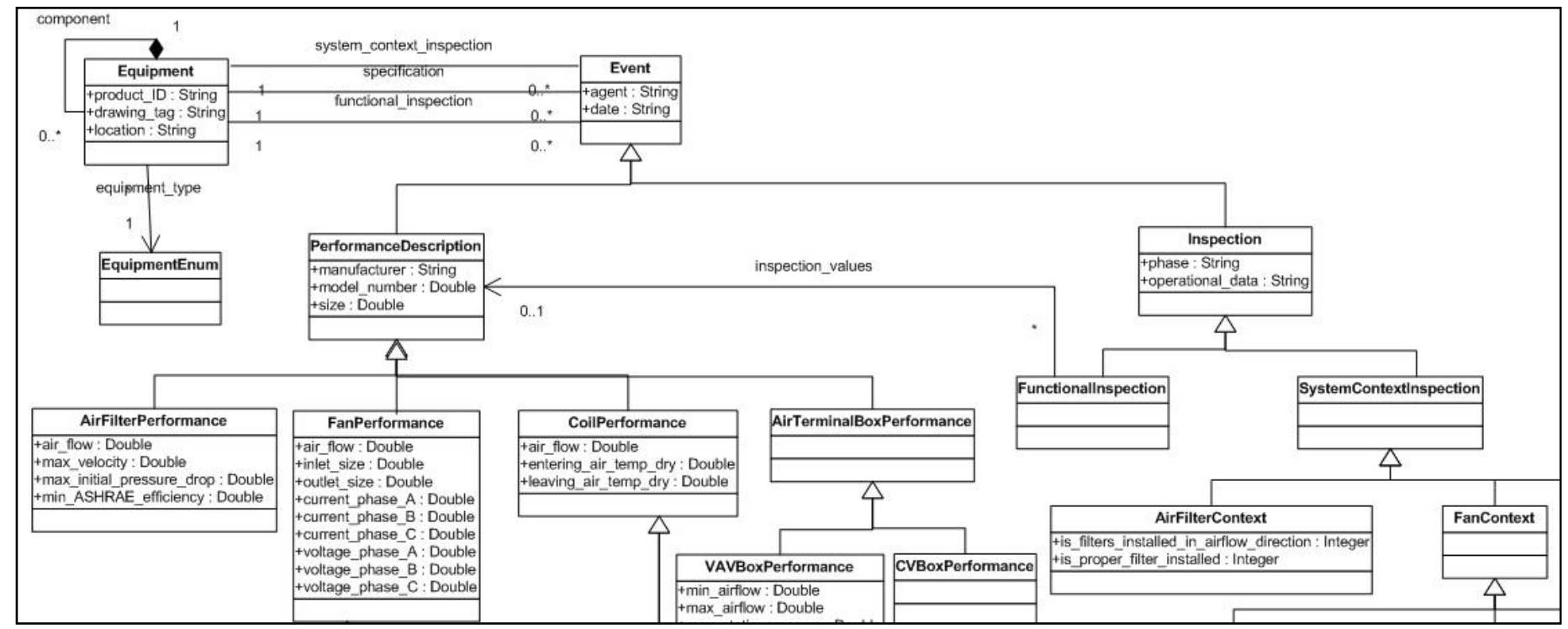

Figure 4. Partial EC Data Model

R2x2 even supports more than $90 \%$ attributes. That value even gets higher when we consider partially matched items. For the classes that are bound to special object or activity, e.g. CentrifugalFanContext and VaneAxialFanPerformance, the quantity of matched items is smaller. Even IFC R2x2, which has improved its HVAC domain greatly, can only fully match less than $20 \% \mathrm{BC}$ attributes, in detail.

\section{PROOF OF CONCEPT PROTOTYPE}

State of the art in current software support for building commissioning has two trends. In the first trend commissioning is seen as an extension to information monitoring or building performance diagnostics tools (Piette et al. 2001, Castro et al. 2003). These tools get information from previously embedded sensors. Through an expert system, this information is evaluated and results are Proceedings of the Fourth International Conference for Enhanced Building Operations, Paris, France, October 18-19, 2004 
represented as excel sheets or data graphs. If there is any deficiency in the system it can be identified from these representations. These tools can only be useful in post-construction equipment tunings or post-occupancy system maintenance.

The second trend of tools focuses more on testing specific equipment such as measuring the exhaust volume of a fan (Rossi et al. 2003). These tools evaluate the performance of specific type of equipment according to their function. They can only be used during the testing phase.

We believe our proof of concept prototype will be different from both of these and support the embedded commissioning process during all of the building lifecycle phases. Neither of these described tools supports the specification or inspection phases of commissioning, two of the three main events of commissioning defined in our EC data model. In our EC process model and EC data model we observed that data evolves with the building lifecycle. During requirement specification every piece of equipment has abstract definitions; whereas in construction and occupancy phases these definitions become detailed.

At this time, we are in the process of mapping the EC data to specific tasks in the EC process model. We are designing the system architecture for our prototype and its functionalities through a group of use cases. In future work, we will implement this as a software application and test it on real time data sets.

\section{SUMMARY OF FINDINGS AND FUTURE WORK}

We have described building commissioning as an important new area of practice and research in the industry. We noted that initially it emerged as the central ingredient of building delivery that is responsible for verifying design intent. We also recognized that in its current form building commissioning is rapidly becoming the choice for performance verification for HVAC systems and LEED certification. Finally, we argued for a computer based technology, called Embedded Commissioning that redefines commissioning as a persistent vehicle for verifying and validating design intent. We pointed out the important implications of this on the design delivery process: decision complexity, system integration and seamless processing. Furthermore, we reported on the work that we have been doing in two areas: (1) eliciting EC data from existing documents and processes in the field, and (2) designing a proof of concept prototype model of HVAC commissioning data and its transformation.

Our future work envisions several additional activities: (1) completing and testing data modeling and exchange applications for the embedded commissioning of HVAC equipment, (2) exploring other areas of EC particularly in the facility management area, and (3) revisiting and refining the larger implications of EC on the entire building lifecycle process, particularly expanding the use of design intent and its tracking as a catalyst for upstream and downstream issues.

\section{REFERENCES}

Akin, O. 1978 "How do architects design?" in J.C. Latombe (ed.), Artificial Intelligence and Pattern Recognition in Computer Aided Design: 65-104. New York: North-Holland Publishing Co.

Akin O. 2001 Ethical Decision Making. Web published class notes, all rights reserved by Omer Akin, School of Architecture, Carnegie Mellon University, Pittsburgh, PA 15213, USA.

Akin, O., Turkaslan-Bulbul, M.T., Brown, S., Kim, E., Akinci, B. \& Garrett, J. 2003. "Comparison of ASHRAE guidelines with building commissioning practice," Proceedings of National Conference on Building Commissioning, May 20-22, California.

American Society of Heating, Refrigerating, \& Air-Conditioning Engineers, Inc. 1996. ASHRAE Handbook HVAC Systems and Equipment, Atlanta, GA.

Castro, N.S., Galler, M.A. \& Bushby, S.T. 2003. "Using the virtual cybernetic building testbed and FDD test shell for FDD tool development." Proceedings of National Conference on Building Commissioning, California, May 20-22.

Davis, G. 1974. "Applying a planned design process and specific research to the planning of offices," in D.H. Carson (ed.), ManEnvironment Interactions: Evaluations and Applications - The State of the Art in Environmental Design Research: 63-89. Stroudsburg, PA: Republished Dowden, Hutchinson and Ross, Inc.

Garrett, J. H., Jr., H. Wang, B. Akinci, O. Akin, M. T. Turkaslan-Bulbul, I. Gursel "Towards Domain-Oriented Semi-Automated Model Matching for Supporting Data Exchange," Proceedings of the ICCCBE, March 2004.

Piette, M.A., Kinney, S. \& Haves, P. 2001 "Analysis of an information management and diagnostic system to improve building operations," Energy and Buildings 33(8): 783-791.

Reilly, J.C. Jr. 1975. Ships of the United States Navy: Christening, Launching and Commissioning, Second Edition. Washington, DC: Naval History Division of the Department of the Navy.

Rossi, T.M., Douglas, J.D. \& Bianchi, M.V.A. 2003. "Evaluating and documenting the performance of unitary HVAC equipment with the Honeywell HVAC service assistant," Proceedings of National Conference on Building Commissioning, 20-22 May, California. 